\title{
Short Circuit Ruggedness of New Generation 1.2 kV SiC MOSFETs
}

\section{Conference Paper}

Author(s):

Kakarla, Bhagyalakshmi (D) Ziemann, Thomas (D); Stark, Roger; Natzke, Philipp; Grossner, Ulrike (D)

Publication date:

2018-10

Permanent link:

https://doi.org/10.3929/ethz-b-000327693

Rights / license:

In Copyright - Non-Commercial Use Permitted

Originally published in:

https://doi.org/10.1109/wipda.2018.8569077 


\title{
Short Circuit Ruggedness of New Generation $1.2 \mathrm{kV} \mathrm{SiC} \mathrm{MOSFETs}$
}

\author{
Bhagyalakshmi Kakarla, Thomas Ziemann, Roger Stark, Philipp Natzke, Ulrike Grossner \\ Advanced Power Semiconductor Laboratory \\ ETH Zurich, Switzerland \\ Email:kakarla@aps.ee.ethz.ch
}

\begin{abstract}
New generations of silicon carbide (SiC) based MOSFETs are commercially available from manufacturers featuring smaller chip size with higher power density demonstrating performance improvement compared to their previous generation counterparts. As the size of the chip is small, the volume available to dissipate energy during short-circuit (SC) like conditions is reduced, leading to increased self-heating of the device. Therefore, the short circuit withstand time (SCWT) is reduced. As a reliability aspect, ruggedness to extreme operating conditions like SC needs to be analyzed for these devices, to improve the design or to design better detection and protection circuits for these MOSFETs when used in specific SC vulnerable applications. In this work, the new third generation $1.2 \mathrm{kV} \mathrm{SiC} \mathrm{MOSFET} \mathrm{from}$ Wolfspeed in a TO-247-4 pin package having a smaller chip size is measured for SC ruggedness. The causes for device failure under different DC-link voltages, gate bias voltages, $\mathrm{SC}$ pulse durations and self-heating behavior are analyzed based on the destructive SC tests performed. The device is measured to have an SCWT of $2 \mu \mathrm{s}$ at a DC-link voltage of $800 \mathrm{~V}$ compared to SCWT of 4.5 $\mu$ s for the second generation $1.2 \mathrm{kV}$ devices with larger chip size and TO-247-3 pin package. The presence of the Kelvin source contact demonstrates higher peak SC currents compared to the same devices without Kelvin source.
\end{abstract}

\section{INTRODUCTION}

$\mathrm{SiC}$ based power devices are foreseen as promising devices in medium to high voltage power electronic systems, because of the material specific advantages such as wide bandgap, high critical electric field, high thermal conductivity, and low intrinsic carrier concentration. With the growing needs for highly efficient power systems, $\mathrm{SiC}$ MOSFETs at various voltage classes are produced by different manufacturers and are commercially available in the voltage ranges of 650 $\mathrm{V}$ to $1.7 \mathrm{kV}$. SiC MOSFETs are applicable in aeronautics, electric vehicles, uninterruptible power supplies, photovoltaic applications, industrial machinery etc.[1]. These are viable alternatives to Si-based IGBTs.

$1.2 \mathrm{kV} \mathrm{SiC} \mathrm{MOSFETs} \mathrm{are} \mathrm{particularly} \mathrm{well} \mathrm{established} \mathrm{both}$ as discrete devices and modules with higher current ratings consisting of multiple $\mathrm{SiC}$ MOSFETs and $\mathrm{SiC}$ schottky diodes. In discrete devices, in order to achieve higher current rating, the area of the chip can be scaled, but when there is a heavy paralleling of cells, there is a necessity to maintain the homogeneity of the cells by means of precise process control. If not, there is a higher risk of failure due to unbalanced current sharing among the cells, decreasing the yield. Efforts to increase the yield while improving the operating performance is a driving force for the development of newer generation of

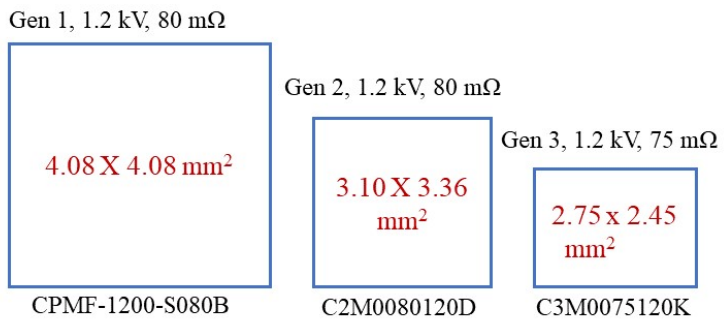

Fig. 1. Trend in chip size (not to scale) with newer generation SiC MOSFETs from Wolfspeed.

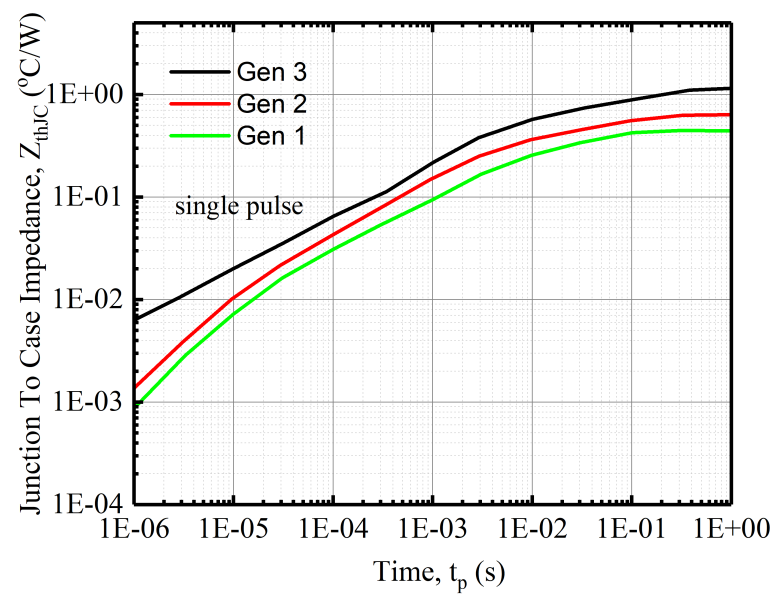

Fig. 2. Junction-to-case thermal impedance of three generation MOSFETs [3][4][5].

SiC MOSFETs with reduced specific on resistance $R_{D S O N}$ and chip sizes leading to higher current densities. Fig. 1 shows the schematic of the trend in chip size with newer generation MOSFETs from Wolfspeed. All the three-generation devices feature a planar gate design [2]. All power devices dissipate a certain energy in the form of heat during their operation. In chips with smaller area, this heat needs to be dissipated in a relatively smaller volume. Fig. 2 shows the thermal impedance of the TO-247 packaged SiC MOSFETs of all 


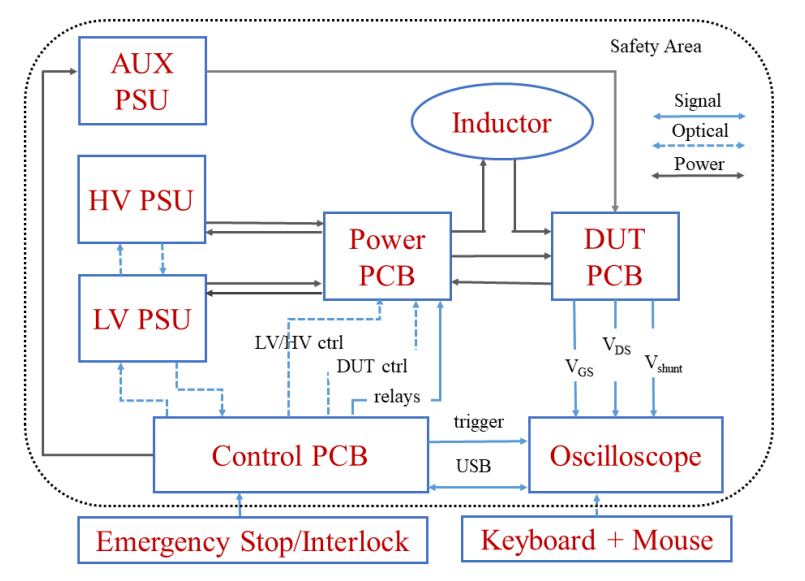

Fig. 3. SC measurement setup schematic.

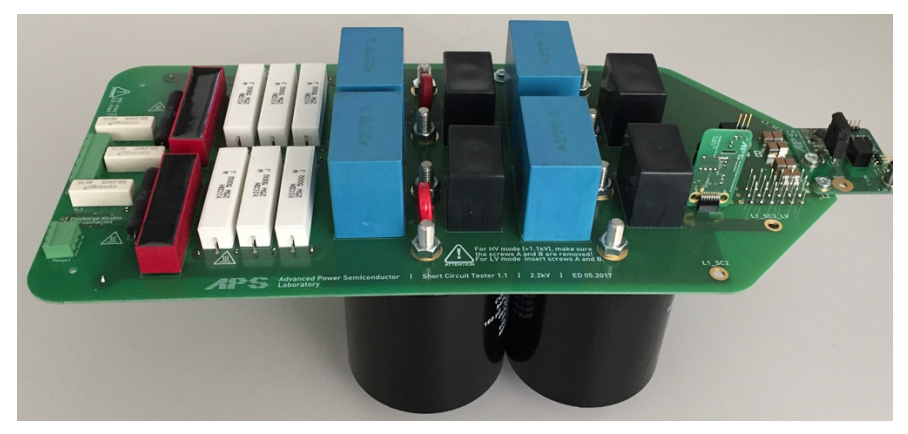

Fig. 4. SC power PCB with capacitor bank.

the three generations with the same voltage rating of $V_{D S}$ $=1.2 \mathrm{kV}$ and $R_{D S O N}$ of $80 \mathrm{~m} \Omega, 80 \mathrm{~m} \Omega$, and $75 \mathrm{~m} \Omega$, respectively, for a current of $I_{D}=20 \mathrm{~A}$. For a given power, the third generation devices having smaller chip area get heated up $\approx 69 \%$ more than their second generation counterparts. When it comes to extreme operating conditions like SC, due to simultaneous high voltage and high current there is very high power dissipation in the device. Therefore, they can heat up much more if the heat is not extracted from the chip efficiently. Hence, the ruggedness during SC of the devices with smaller chip sizes needs to be thoroughly assessed to ensure reliability of these devices in mission profiles where a $\mathrm{SC}$ like condition is possible. In this work, two generation of MOSFETs with different chip sizes from Wolfspeed are tested and compared in terms of SC ruggedness. The second generation devices (here Gen 2) tested are C2M0080120D, TO-247-3 packaged having larger chip-size (active area of $\approx 6.4 \mathrm{~mm}^{2}$ ) and a specific $R_{D S O N}$ of $5.6 \mathrm{~m} \Omega \mathrm{cm}^{2}$, while the third generation devices (here Gen 3 ) are TO-247-4 packaged having smaller chip size (active area of $\approx 4 \mathrm{~mm}^{2}$ ) and a specific $R_{D S O N}$ of $2.6 \mathrm{~m} \Omega \mathrm{cm}^{2}$. The Gen 3 devices are new on the market demonstrating excellent efficiency in on-state with low $R_{D S O N}$ and efficient switching with a Kelvin source contact included in the TO-247 [6]. In addition, Gen 3 devices are operated at a lower gate bias $V_{G S}=15 \mathrm{~V} /-4 \mathrm{~V}$ (IGBT-like

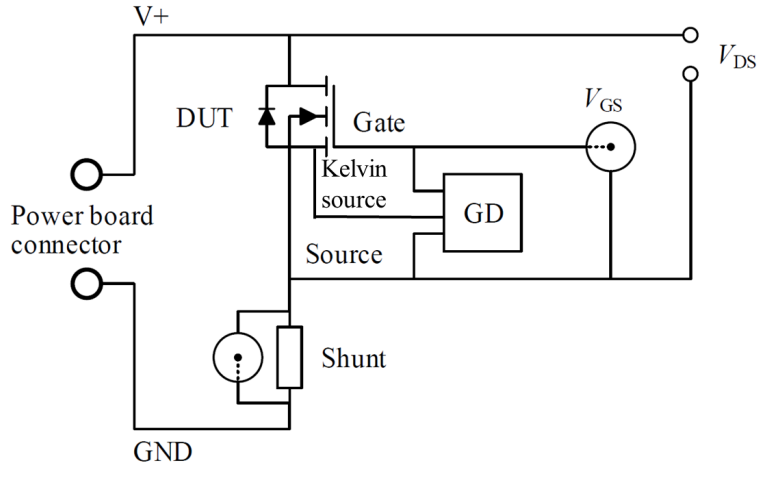

Fig. 5. DUT PCB schematic.

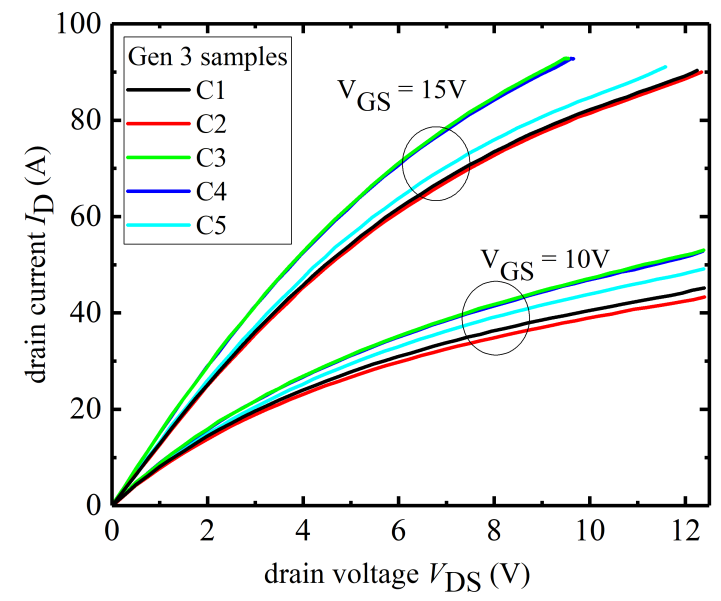

Fig. 6. On-state characteristics measured for 5 samples of Gen 3 MOSFET at room temperature, $\mathrm{T}=295 \mathrm{~K}$.

on-state gate bias).

The SC performance and failure mechanisms of discrete $\mathrm{SiC}$ MOSFET devices in the $1.2 \mathrm{kV}$ voltage class are presented in [7][8][9]. The cause for failure analysis can be used to improve the design strategies which focus on improving SC robustness with a minimum compromise on the nominal operating performance [10][11]. However, Gen 3 devices are new and the SC performance of these devices is not reported in literature. Therefore, this work aims to analyse the device behaviour of such small chips under SC stress, the impact of the Kelvin source contact on SC ruggedness, and to present the failure modes observed.

\section{Measurement Setup}

The main idea of the SC test is to subject the device under test (DUT) to the entire operational DC-link voltage in order to emulate the condition of hard switching fault. The schematic of the SC measurement setup is shown in Fig. 3. It consists of two main power supply units (PSU). These PSUs are used to charge a capacitor bank to the desired DC-link voltage for testing. The capacitors are mounted on a printed circuit 


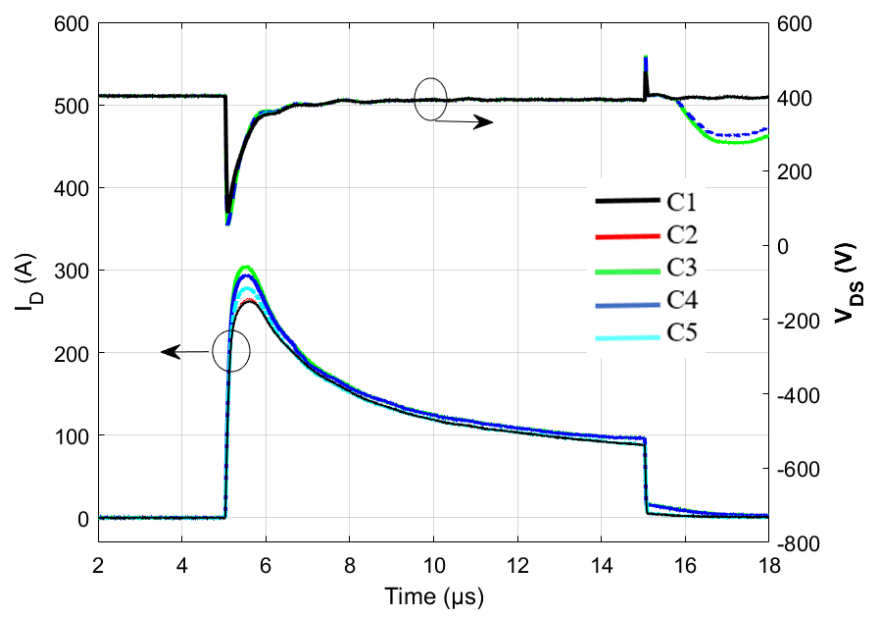

Fig. 7. Influence of performance spread on $\mathrm{SC}$ at $V_{D S}=400 \mathrm{~V}, V_{G S}=15$ V/ $-4 \mathrm{~V}, t_{s c}=10 \mu \mathrm{s}$ observed for the 5 samples of Gen 3 MOSFETs with on-state performance as shown in Fig. 6.

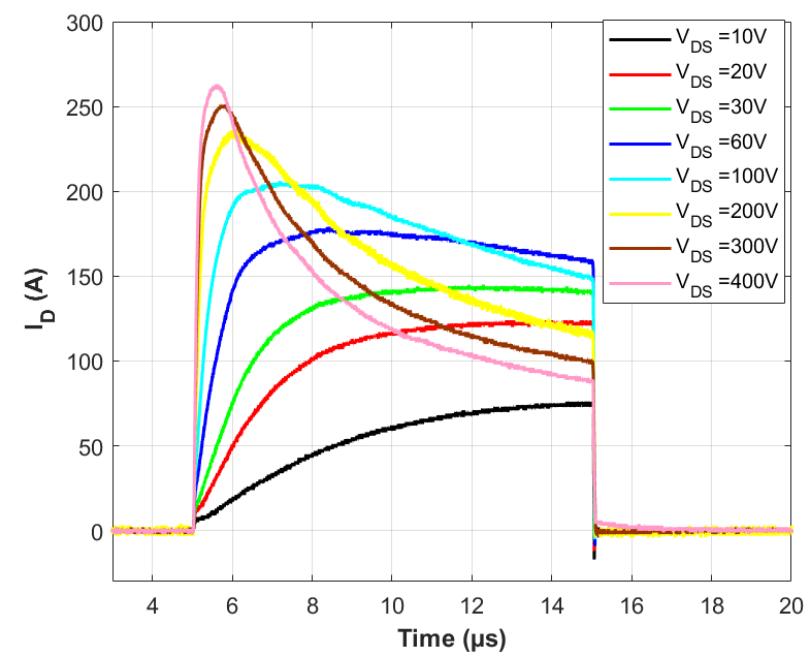

Fig. 8. Influence of $V_{D S}$ measured for Gen 3 MOSFET at $t_{s c}=10 \mu \mathrm{s}, V_{G S}$ $=15 \mathrm{~V} /-4 \mathrm{~V}, \mathrm{~T}=295 \mathrm{~K}$.

board (PCB). The power PCB with mounted capacitors, fast discharge resistors, relays and metal-oxide varistors (MOV) to protect the capacitor bank from over voltages is shown in Fig. 4. The DUT PCB is designed based on Fig. 5, which has an on-board gate driver (GD) (UCC27531 of Texas Instruments) to achieve low parasitic inductance and, hence, faster gate control. It features separate turn-on and turn-off output voltages. The power PCB and DUT are controlled by a control PCB. The control PCB is connected to the oscilloscope (Keysight DSO, $500 \mathrm{MHz}, 10 \mathrm{GS} / \mathrm{s}, 10 \mathrm{bit}$ ). The SC currents of the devices can go as high as $300 \mathrm{~A}$ in the beginning of the SC and reach more than 1000 A during failures. In order to measure the currents accurately, a coaxial shunt (SBNC-2-025 of TM), with a low resistance of $25 \mathrm{~m}$ and high energy rating of $40 \mathrm{~J}$ is used.

\section{RESUlTS AND DISCUSSION}

The static output characteristics measured at room temperature $\mathrm{T}=295 \mathrm{~K}$ of 5 samples of Gen 3 devices at a gate bias of $V_{G S}=10 \mathrm{~V}$ and $V_{G S}=15 \mathrm{~V}$ are shown in the Fig. 6. It can be seen that at nominal operating current of $I_{D}=$ 20 A with $V_{G S}=15 \mathrm{~V}$, there is minimal spread in $R_{D S O N}$ whereas at higher currents, i.e. $I_{D}=80 \mathrm{~A}$, there is a significant spread. The influence of such a spread and the influence of test conditions like $V_{D S}, V_{G S}$, and $t_{s c}$ on the SC behaviour of Gen 3 MOSFETs is subsequently analysed.

\section{A. SC behaviour of SiC MOSFETS}

The changes in $I_{D}$ during an $\mathrm{SC}$ event can be reviewed based on Fig. 7. At $V_{D S}=400 \mathrm{~V}$, with $V_{G S}=15 \mathrm{~V} /-4 \mathrm{~V}$ and $t_{s c}=10 \mu \mathrm{s}$, the current $I_{D}$ rises rapidly at SC turnon when $V_{G S}$ switches from $-4 \mathrm{~V}$ to $15 \mathrm{~V}$ at time $5 \mu \mathrm{s}$. Depending on the stray inductances in the circuit $L_{s t r a y}$, gate resistance $R_{g}$, and gate capacitances $C_{G D}$ and $C_{G S}$ of the device, the rate of change of current (di/dt) at the SC turnon transient causes an overshoot current [12]. After such a transient and reaching a peak current, $I_{D}$ starts falling due to the high power dissipated which causes self-heating of the device. This phenomenon reduces the bulk mobility, leading to a reduction in drain current $I_{D}$. With increasing SC time, the device continues to heat up. Depending on the cell design, the electric fields, and lattice temperature in the device, a positive slope in current can be observed in longer pulses. The increase in current maybe due to triggering of the parasitic BJT when the cell design is weak, or intrinsic carrier generation due to very high lattice temperatures [13][14]. These currents can be called leakage currents. When the device is forced to turnoff from SC with the applied $V_{G S}$, only the channel current is switched off but the leakage current is not off. Depending on the amount of current and the underlying mechanism that generates the carriers for the leakage current, the device gets degraded or fails.

1) Influence of performance spread on SC behavior:: The performance spread at higher currents leads to different peak SC drain currents at SC turn-on which can be seen in Fig. 7. For a given $V_{D C}=400 \mathrm{~V}$, the devices $\mathrm{C} 3$ and $\mathrm{C} 4$ show $40 \mathrm{~A}$ and $50 \mathrm{~A}$ higher peak currents, respectively, compared to C1. At SC turn-off, these devices demonstrate high leakage currents or tail currents in the order of $I_{D}=25$ $30 \mathrm{~A}$. The presence of such high leakage currents at turn-off leads to loss of gate control and device degradation. Therefore, the device loses its voltage blocking capability leading to failure after SC turn-off which can be seen as voltage drop in Fig.7 at $16 \mu$ s. When paralleling MOSFETs in a module, it is therefore necessary to choose the devices with minimum performance spread [15][16][17] not only in the operating current regime, but also in higher current regime to ensure better SC ruggedness of the module.

2) Influence of $V_{D S}$ on $S C:: 1.2 \mathrm{kV}$ rated devices are normally operated at a typical DC-link voltage of $V_{D S}=$ $800 \mathrm{~V}$ (2/3rd of rated voltage). As the applied $V_{D S}$ increases, for a given $V_{G S}$, during $\mathrm{SC} I_{D}$ increases rapidly as seen in 


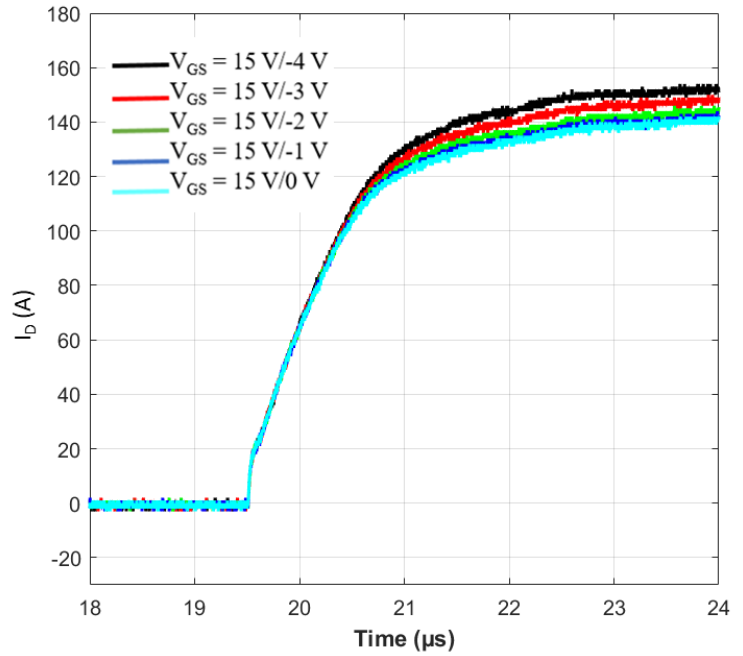

Fig. 9. Influence of negative $V_{G S}$ on SC currents at turn on at $V_{D S}=200$ $\mathrm{V}, t_{s c}=10 \mu \mathrm{s}, \mathrm{T}=295 \mathrm{~K}$ with Kelvin source contact.

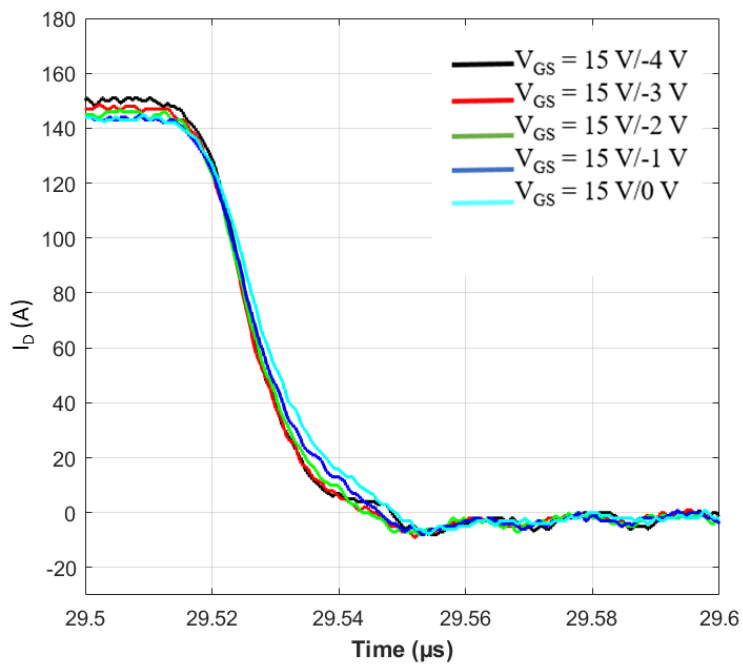

Fig. 10. Influence of negative $V_{G S}$ on SC currents at turn off at $V_{D S}=200$ $\mathrm{V}, t_{s c}=10 \mu \mathrm{s}, \mathrm{T}=295 \mathrm{~K}$ with Kelvin source contact.

Fig. 8. Therefore, there is also a rapid rise in temperature due to very high power dissipated $\left(V_{D S} * I_{D}\right)$. The Gen 3 MOSFETs, when tested with the nominal $V_{G S}=15 \mathrm{~V} /-4 \mathrm{~V}$, could withstand an SC pulse of $t_{s c}=10 \mu \mathrm{s}$ at $V_{D S}=400 \mathrm{~V}$, but at $V_{D S}=600 \mathrm{~V}$, they could survive only upto $t_{s c}=4-6$ $\mu \mathrm{s}$, and at $V_{D S}=800 \mathrm{~V}$, they could only hardly survive $t_{s c}=$ $2 \mu \mathrm{s}$.

3) Influence of turn-on $V_{G S}$ on $S C::$ Considering the turnon $V_{G S}$ : The Gen 3 MOSFETs are operated efficiently at an IGBT like bias of turn-on $V_{G S}=15 \mathrm{~V}$, compared to the Gen 2 devices for which $V_{G S}=20 \mathrm{~V}$. With higher gate bias voltage, the inversion channel of the MOSFET is strengthened with lower channel resistance because of higher transconductance. Hence, the higher the $V_{G S}$, the higher the on-state current $I_{D}$ and therefore, also the higher the SC currents for a given

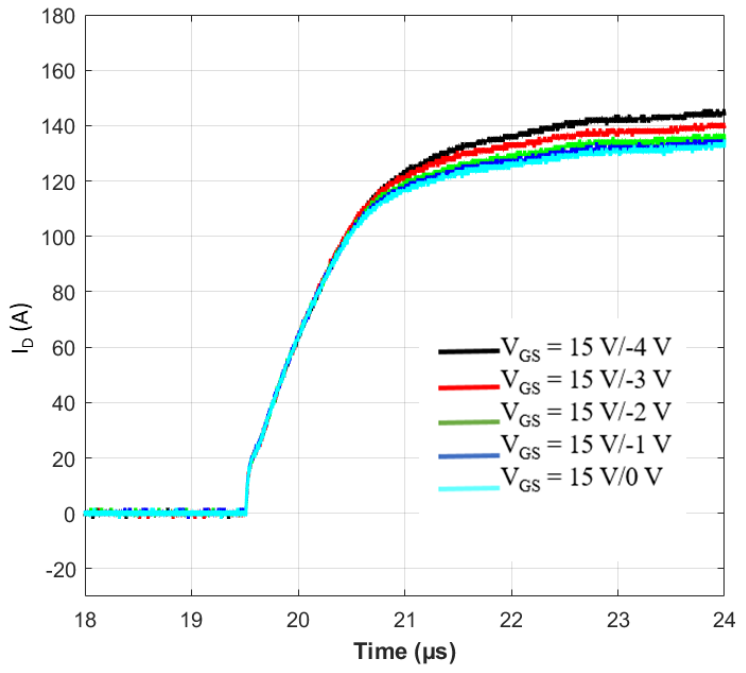

Fig. 11. Influence of negative $V_{G S}$ on SC currents at turn on at $V_{D S}=200$ $\mathrm{V}, t_{s c}=5 \mu \mathrm{s}, \mathrm{T}=295 \mathrm{~K}$ without Kelvin source contact.

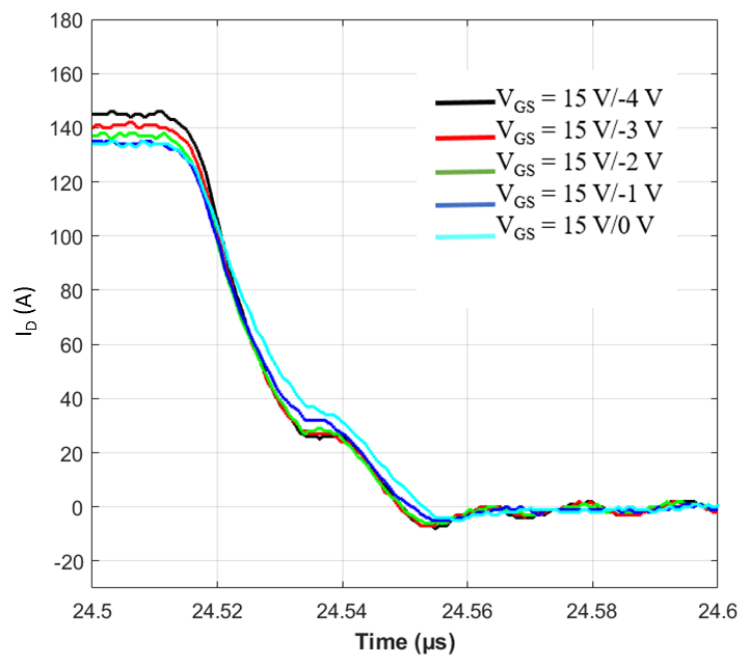

Fig. 12. Influence of negative $V_{G S}$ on SC currents at turn off at $V_{D S}=200$ $\mathrm{V}, t_{s c}=5 \mu \mathrm{s}, \mathrm{T}=295 \mathrm{~K}$ without Kelvin source contact.

$V_{D S}$, thus limiting the SC duration. However, depending on the operating temperature, MOSFETs can also be operated at lower gate bias voltage than the nominal operating gate bias with a trade-off on the on-state performance for applications that require better SC ruggedness.

4) Influence of turn-off $V_{G S}$ on $S C::$ As shown for the influence of turn-off $V_{G S}$ in Fig. 9 and Fig. 10, Gen 3 MOSFETs show higher peak SC currents with higher negative bias and faster turn-off. Such high peak currents due to negative gate bias were not observed in Gen 2 MOSFETs. This is consistently observed in all the 15 samples tested and at all $V_{D S}$ conditions. The reason for this behavior can be attributed to threshold voltage change and trap distribution in the MOS interface. It is only speculated that there is a threshold voltage shift occuring with the higher negative gate 

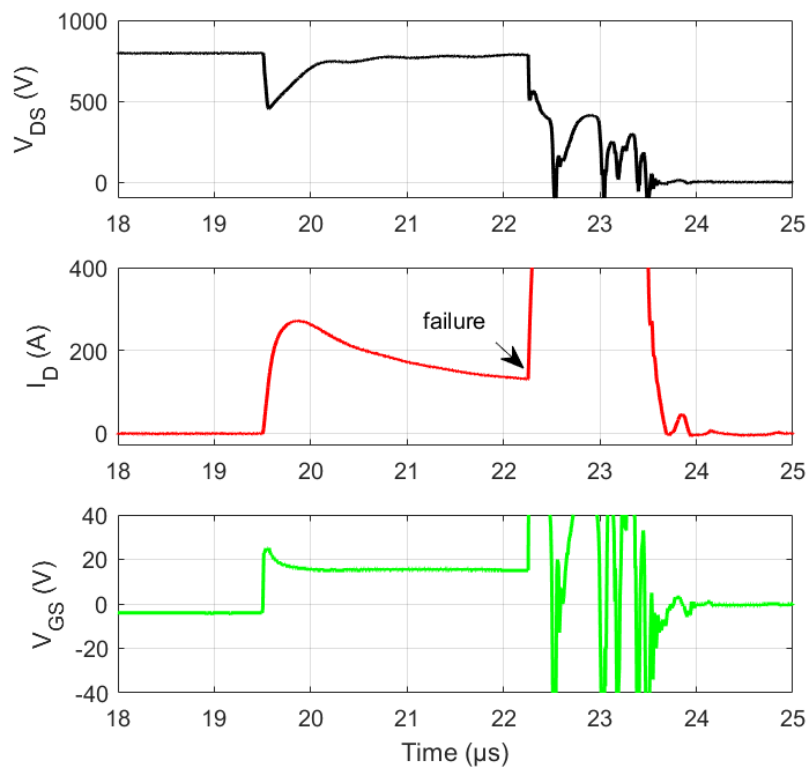

Fig. 13. SC behavior of a Gen $3 \mathrm{SiC}$ MOSFET during a SC test at $V_{D S}=$ $800 \mathrm{~V}, V_{G S}=15 \mathrm{~V} /-4 \mathrm{~V}, \mathrm{~T}=295 \mathrm{~K}, t_{s c}=2 \mu \mathrm{s}$.

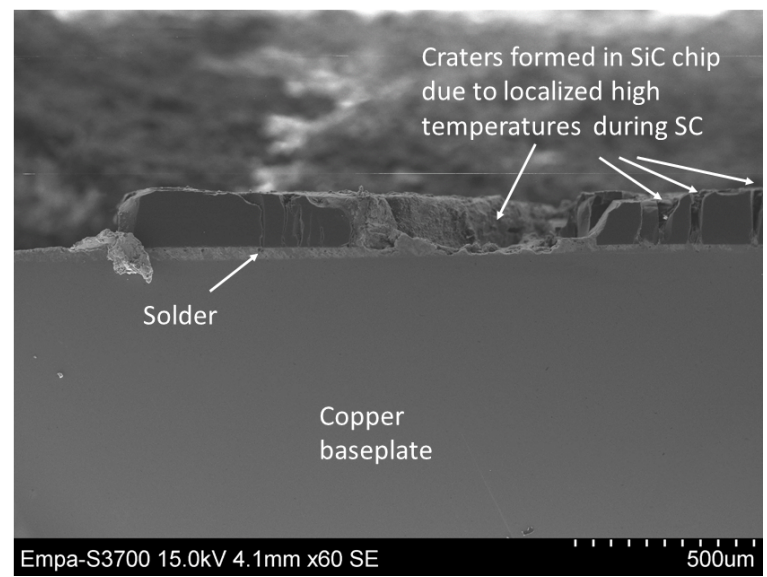

Fig. 14. SEM cross-section image of the failed TO-247 Gen 3 MOSFET showing the high temperature effect on the SiC chip post SC duration of $t_{s c}$ $=2.2 \mu \mathrm{s}$.

bias causing this high peak SC currents. However, it needs further analysis based on the exact internal device structure, MOS interface, and the internal device capacitances.

5) Influence of the Kelvin source contact on SC:: Gen 3 MOSFETs rated for $1.2 \mathrm{kV}$ are available only as TO-2474 pin packages with an extra source pin acting as a Kelvin source contact compared to Gen 2 devices with TO-247-3 pin package. With the Kelvin source contact, the source inductance induced gate voltage modulation at the switching transients is minimized. Therefore, the devices with a Kelvin source contact turns on faster. The Gen 3 devices were also tested without Kelvin source contact at the $V_{D S}=200 \mathrm{~V}$ (shown in Fig. 11 and Fig. 12). Comparing Fig. 9 and Fig. 11, it can be seen that for a given sample there is $\mathrm{a} \approx 10 \mathrm{~A}$ higher peak SC current
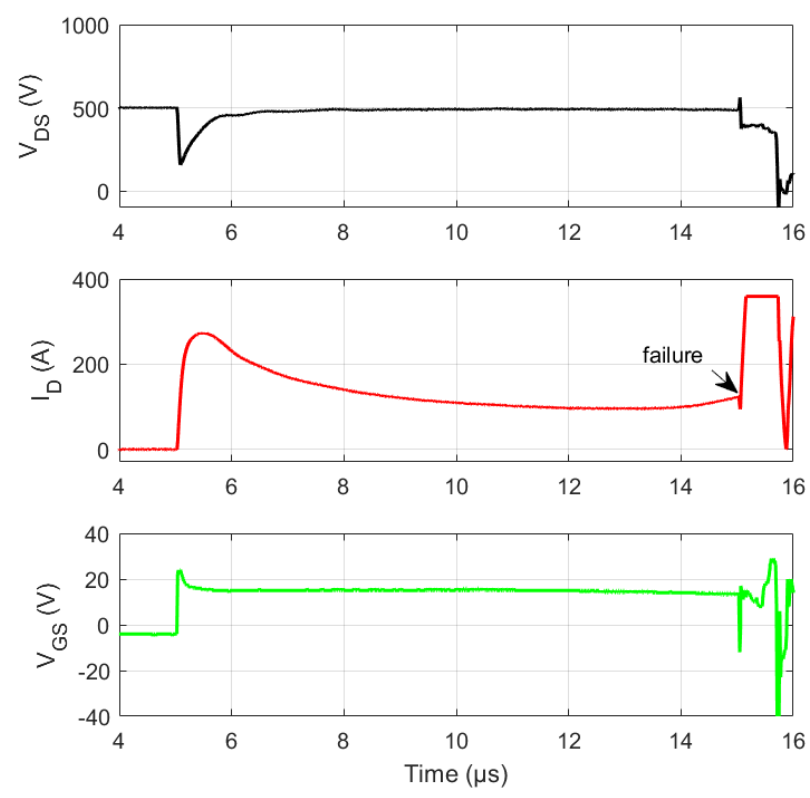

Fig. 15. SC behavior of a Gen $3 \mathrm{SiC}$ MOSFET during a $\mathrm{SC}$ test at $V_{D S}=$ $500 \mathrm{~V}, V_{G S}=15 \mathrm{~V} /-4 \mathrm{~V}, \mathrm{~T}=295 \mathrm{~K}, t_{s c}=10 \mu \mathrm{s}$. The device failed at $10 \mu \mathrm{s}$ when trying to turn-off.

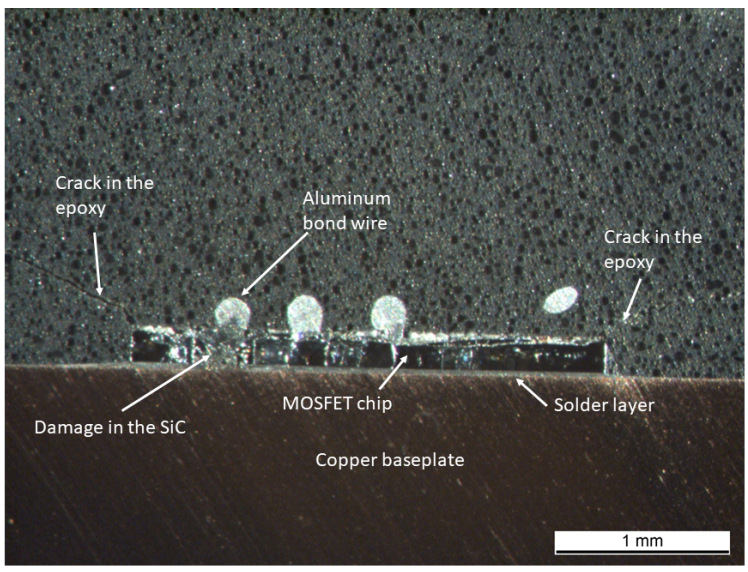

Fig. 16. Optical microscope image of the failed Gen 3 MOSFET chip showing degradation in the $\mathrm{SiC}$ material, molten aluminum at the contact and crack in the epoxy of the TO-247 package.

with Kelvin source for a given $V_{G S}$. This increases the peak power compared to SC turn-on without Kelvin source. Also, the SC turn-off is faster with Kelvin source [18] compared to devices without Kelvin source. This is because, at turn-off there is a negative di/dt between drain-source terminal leading to a voltage drop across the source inductance. This adds up to gate drive voltage delaying the turn-off of the device if there is no Kelvin source.

\section{B. SC failure analysis of SiC MOSFETS}

Fig. 13 shows the $I_{D}, V_{D S}$ and $V_{G S}$ plot of a sample that failed during the SC with $V_{G S}$ at on-state after an SC stress 


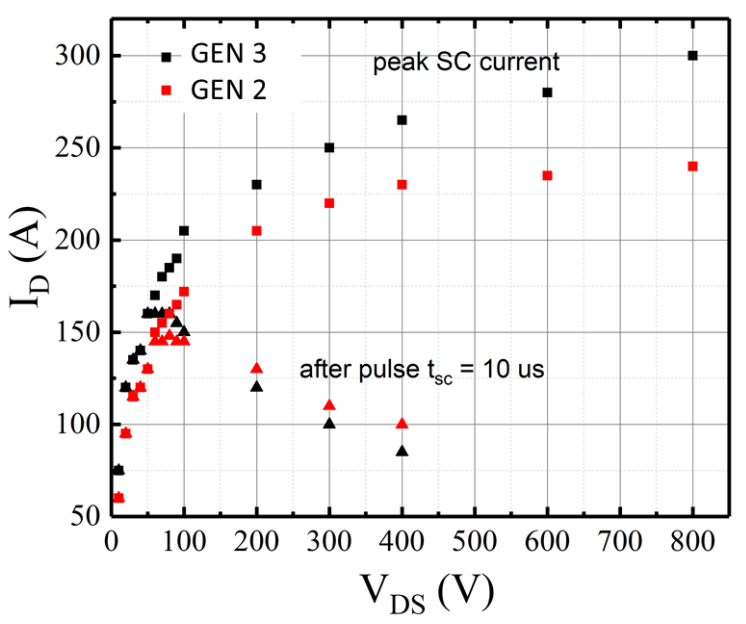

Fig. 17. SC currents of Gen 2 and Gen 3 MOSFETs with different $V_{D S}$ for a pulse of $t_{s c}=10 \mu$ s tested at $\mathrm{T}=295 \mathrm{~K}$ with operating $V_{G S}=20 \mathrm{~V} /-5 \mathrm{~V}$ (for Gen 2) and $V_{G S}=15 \mathrm{~V} /-4 \mathrm{~V}$ (for Gen 3).

with $V_{D S}=800 \mathrm{~V}, t_{s c}=3 \mu \mathrm{s}, V_{G S}=15 \mathrm{~V} / 4 \mathrm{~V}$. The device had a peak power of $193 \mathrm{~kW}$ during SC and the critical energy EC at failure was $0.25 \mathrm{~J}$ at $t_{s c}=2.2 \mu \mathrm{s}$. The device failed during on state with sudden rise in gate voltage. The device blew up lifting off the epoxy on the top side of the TO-package. The remaining TO package was cut at $3 \mathrm{~mm}$ from the contact edge to see the cross section under SEM. Fig. 14 shows the cross-section SEM image in which the $\mathrm{SiC}$ appears to have evaporated at various areas leaving behind craters because of localised extreme temperatures in the chip. Another sample tested with DC-link voltage $V_{D S}=800 \mathrm{~V}, V_{G S}=15 \mathrm{~V} /-4 \mathrm{~V}$, $t_{s c}=2 \mu$ s turned off the SC current but failed immediately after turn off. A sample tested with a lower DC-link voltage of $V_{D S}=500 \mathrm{~V}$ and a longer SC pulse of $t_{s c}=10 \mu \mathrm{s}$ has an $\mathrm{SC}$ behavior as shown in Fig. 15. The positive slope in current is observed after $t_{s c}=9 \mu \mathrm{s}$, then at turn-off a dip in $I_{D}$ and a small peak in $V_{D S}$ is seen but the device failed subsequently with a sudden rise of $I_{D}$ signifying the loss of gate control. All the samples tested with longer pulses and lower $V_{D S}$ failed with the signature of high gate source leakage currents after SC turn-off[19]. Devices that failed with high gate leakage currents showed soft failure with no visible damage of the TO247 package. However, on slicing the package of a sample that had a soft failure and visualizing under an optical microscope, the degradation in the chip and molten aluminum contact is observed (see Fig. 16). A crack in the epoxy at the edges of the chip is visible which can be correlated to thermal stress propagation from the chip.

\section{Comparison of Gen 2 and Gen 3 MOSFETs during SC}

For an operating current of $I_{D}=20 \mathrm{~A}$, Gen 2 has an $R_{D S O N}$ of $80 \mathrm{~m} \Omega$ at the recommended gate bias of $V_{G S}=$ $20 \mathrm{~V}$ while the Gen 3 MOSFET has an $R_{D S O N}$ of $75 \mathrm{~m} \Omega$ at the recommended $V_{G S}=15 \mathrm{~V}$. When subjected to SC stress with various DC-link voltages starting from $V_{D S}=10 \mathrm{~V}$ to $V_{D S}=800 \mathrm{~V}$ with $t_{s c}=10 \mu \mathrm{s}$, the peak current at SC turn- on of the Gen 3 MOSFET for any given DC-link voltage is always higher than the Gen 2 MOSFET as plotted in Fig. 17. The current after $t_{s c}=10 \mu \mathrm{s}$ is only plotted up to $V_{D S}=$ $400 \mathrm{~V}$ as none of the devices survived beyond this voltage for $10 \mu \mathrm{s}$. Although the Gen 3 MOSFET is operated with a lower gate turn-on voltage, higher peak currents lead to higher power dissipation. Thus, under same packaging conditions, the SCWT of Gen 3 is less than Gen 2 and is limited mainly by self-heating[20].

\section{CONCLUSION}

This work presents the SC behavior of $1.2 \mathrm{kV}, 75 \mathrm{~m} \Omega$, TO247-4 pin packaged C3M0075120K SiC MOSFETs. The peak SC drain currents of the Gen 3 devices are higher than that of the Gen 2 devices for any given $V_{D S}$ and show higher power dissipation. The SCWT of C3M0075120K device is measured to be SCWT $=2 \mu$ s at nominal operating DC-link voltage of $V_{D S}=800 \mathrm{~V}$ with a gate of $V_{G S}=15 \mathrm{~V} /-4 \mathrm{~V}$ compared to $\mathrm{SCWT}=4.5 \mu$ s of the Gen 2 device. It is crucial for the system designers to design protective systems that can respond within $2 \mu \mathrm{s}$ in order to avoid catastrophic destruction of the system. In the majority of the samples tested, failure is originating in the die due to high temperatures. As SC is transient and the die encounters very high temperatures at the junction close to the surface, the heatsink in the drain-side of the TO-247 package is not sufficient to extract the heat out of the die. Failure in the packaged die due to high temperature effects is evident from the cross-section images. Advanced packaging methodologies to cool the die from the top side, quick, compact SC detection and protection circuits, as well as innovative cell design solutions to preserve efficiency at nominal operating conditions while limiting the current at higher drain bias can help to achieve reliability of smaller dies in applications vulnerable to SC like conditions.

\section{ACKNOWLEDGMENT}

The authors greatly acknowledge the ETH Zurich Foundation for their financial support. Our acknowledgment goes to EMPA for using the instrumental resources to get the SEM images.

\section{REFERENCES}

[1] M. Furuhashi, S. Tomohisa, T. Kuroiwa, and S. Yamakawa, Practical applications of SiC-MOSFETs and further developments, Semiconductor Science and Technology, 2016.

[2] J. Casady, B. Development, P. Manager, and M. B. Hayes, Impact of Ultra-Low On-Resistance SiC MOSFETs On Electric Vehicle DriveTrain, Power Electronics Europe, 2017.

[3] CREE Datasheet, CMF20120D, Z-FET ${ }^{T} M$ Silicon Carbide MOSFET NChannel Enhancement Mode MOSFET. ,2012.

[4] CREE Datasheet, C2M0080120D, C2M $M^{T M}$ Silicon Carbide MOSFET N-Channel Enhancement Mode Bare Die, ,2015.

[5] CREE Datasheet, C3M0075120K, C3M $M^{T M}$ Silicon Carbide MOSFET NChannel Enhancement Mode MOSFET, ,2017.

[6] C. Bodeker, E. Ayerbe, and N. Kaminski, Impact of a Kelvin Source Connection on Discrete High Power SiC-MOSFETs, Materials Science Forum, 2018.

[7] C. Chen , D. Labrousse, S. Lefebre, M. Petit, C. Buttay, H. Morel, Robustness in short-circuit Mode of SiC MOSFETs, PCIM Power Electronics Conference, 2015. 
[8] G. Kampitsis, S. Papathanassiou, and S. Manias, Comparative evaluation of the short-circuit withstand capability of $1.2 \mathrm{kV}$ silicon carbide $(\mathrm{SiC})$ power transistors in real life applications, Microelectronics Reliability, 2015.

[9] B. Kakarla, T. Ziemann, S. Nida, E. Doenni, and U. Grossner, Planar to Trench: Short Circuit Capability Analysis of $1.2 \mathrm{kV} \mathrm{SiC} \mathrm{MOSFETs,}$ Materials Science Forum, 2018.

[10] G. Romano, A. Fayyaz, M. Riccio, L. Maresca, G. Breglio, A. Castellazzi, and A. Irace, A comprehensive stdy of short-circuit ruggedness of silicon carbide power MOSFETs, IEEE Journal of Emerging and Selected Topics in Power Electronics, 2016.

[11] T. Shoji, A. Soeno, H. Toguchi, S. Aoi, Y. Watanabe, and H. Tadano, Short-circuit Capability of SiC Power MOSFETs, 2014.

[12] B. Kakarla, S. Nida, J. Mueting, T. Ziemann, I. Kovacevic-Badstuebner, and U. Grossner, Trade-off analysis of the p-base doping on ruggedness of SiC MOSFETs, Microelectronics Reliability, 2017.

[13] A. Kadavelugu, and E. Aeloiza, Short circuit performance of multi-chip SiC MOSFET modules, IEEE 5th Workshop on Wide Bandgap Power Devices and Applications (WiPDA), 2017.

[14] H. Du, P. D. Reigosa, F. Iannuzzo, and L. Ceccarelli, Short-Circuit Tests of $1.2 \mathrm{kV} \mathrm{SiC} \mathrm{MOSFET} \mathrm{Power} \mathrm{Module,} \mathrm{CORPE} \mathrm{Annual} \mathrm{Symposium,}$ 2018.

[15] J. Mueting, N. Schneider, T. Ziemann, R. Stark, and U. Grossner, Exploring the behavior of parallel connected SiC power MOSFETs influenced by performance spread in circuit simulations, Conference Proceedings IEEE Applied Power Electronics Conference and Exposition APEC, 2018

[16] V. D. Karaventzas, M. Nawaz, and F. Iannuzzo, Reliability Assessment of SiC Power MOSFETs From The End User s Perspective, Energy Conversion Congress and Exposition (ECCE), 2016.

[17] A. J. Lelis, R. Green, and D. B. Habersat, High-Temperature Reliability of SiC Power MOSFETs, IEEE Materials Science Forum, 2011.

[18] A. Gaito, R. Scollo, G. Panebianco and A. Raciti, Impact of the sourcepath parasitic inductance on the MOSFET commutations, IEEE Energy Conversion Congress and Exposition (ECCE), 2012.

[19] B. J. Nel and S. Perinpanayagam, A Brief Overview of SiC MOSFET Failure Modes and Design Reliability, Procedia CIRP, 2017.

[20] T. Terashima, Superior performance of SiC power devices and its limitation by self-heating, Technical Digest - International Electron Devices Meeting, IEDM, 2017. 